\title{
Modulation of Surface-Initiated ATRP by Confinement: Mechanism and Applications
}

\author{
Edmondo M. Benetti,*(i) Chengjun Kang, Joydeb Mandal, Mohammad Divandari, \\ and Nicholas D. Spencer*(1)
}

Laboratory of Surface Science and Technology, Department of Materials, ETH Zurich, Vladimir-Prelog-Weg 5, CH-8093 Zürich, Switzerland

Supporting Information

ABSTRACT: The mechanism of surface-initiated atom transfer polymerization (SI-ATRP) of methacrylates in confined volumes is systematically investigated by finely tuning the distance between a grafting surface and an inert plane by means of nanosized patterns and micrometer thick foils. The polymers were synthesized from monolayers of photocleavable initiators, which allow the analysis of detached brushes by sizeexclusion chromatography (SEC). Compared to brushes
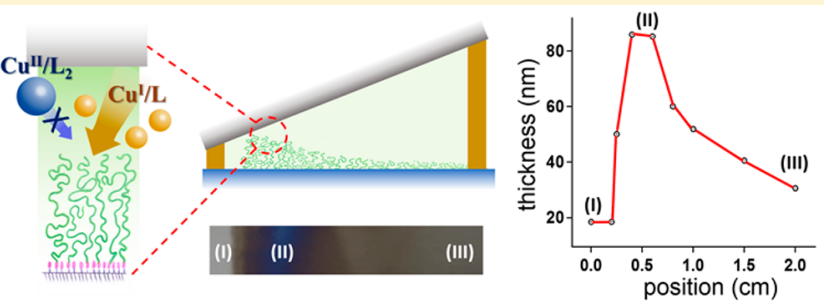
synthesized under "open" polymerization mixtures, nearly a 4-fold increase in brush molar mass was recorded when SI-ATRP was performed within highly confined reaction volumes. Correlating the SI-ATRP of methyl methacrylate (MMA), with and without "sacrificial" initiator, to that of lauryl methacrylate (LMA) and analyzing the brush growth rates within differently confined volumes, we demonstrate faster grafting kinetics with increasing confinement due to the progressive hindering of $\mathrm{Cu}^{\mathrm{II}}$-based deactivators from the brush propagating front. This effect is especially noticeable when viscous polymerization mixtures are generated and enables the synthesis of several hundred nanometer thick brushes within relatively short polymerization times. The faster rates of confined SI-ATRP can be additionally used to fabricate, in one pot, precisely structured brush gradients, when volume confinement is continuously varied across a single substrate by spatially tuning the vertical distance between the grafting and the confining surfaces.

\section{INTRODUCTION}

The fabrication of chain-end-tethered polymer assemblies or polymer "brushes"1,2 within highly confined volumes has been the subject of considerable attention by researchers from different fields of chemistry and materials science. Polymer brushes have been synthesized by surface-initiated controlled radical polymerization (SI-CRP) ${ }^{3}$ within the cavities of nano/ mesoporous supports to yield organic-inorganic hybrids, ${ }^{4-6}$ to control the transport of liquids and the separation of biomolecules across nanostructured, inorganic membranes, ${ }^{7-14}$ and to design synthetic analogues of cellular membranes, with the aim of tuning the trafficking of ionic species between separate compartments. ${ }^{15}$ Additionally, SI-CRP has been performed from the inner walls of microchannels ${ }^{16,17}$ and subsequently employed to fabricate reactors featuring a high loading of polymer-brush-supported organic and inorganic catalysts. ${ }^{18,19}$ Despite the variety of fabrications involving SICRP within confined volumes, featuring micro- or nanostructured environments, the influence on the polymerization mechanism of physical boundaries with typical dimensions ranging from the micrometer scale down to a few hundred nanometers has never been systematically addressed. Several reports focusing on the synthesis of polymer brushes from bidimensional nanopatterns have described how the conformation of densely grafted chains is determined by the size of the initiating support. ${ }^{20}$ However, little has been reported on the influence of confined volumes on the grafting process. Especially for SI-CRP-where the initiating functions are already confined to a planar surface-we demonstrate that the presence of additional physical constraints markedly influences the entire grafting process, when compared to an "open" polymerization medium where monomers and catalysts can freely diffuse and mix together.

A complete understanding of confinement effects on SI-CRP holds the potential of providing a fundamental advance in this widely applied surface-modification technique and of expanding the application of polymer brushes to increasingly sophisticated micro- and nanofabricated systems.

We particularly focus on surface-initiated atom transfer polymerization (SI-ATRP) - a highly versatile SI-CRP method with a well-controlled mechanism and compatibility with a wide range of monomers and solvents. ${ }^{21-23}$ The systematic investigation of the effects of volume confinement on SIATRP involves tackling a number of experimental and technical challenges, which have hitherto impeded a complete understanding: (i) it is challenging to precisely evaluate polymerbrush thickness within spatially confined environments, as in the cases of nanotubes or nanoporous inorganic substrates; (ii)

Received: May 3, 2017

Revised: June 20, 2017

Published: July 19, 2017 

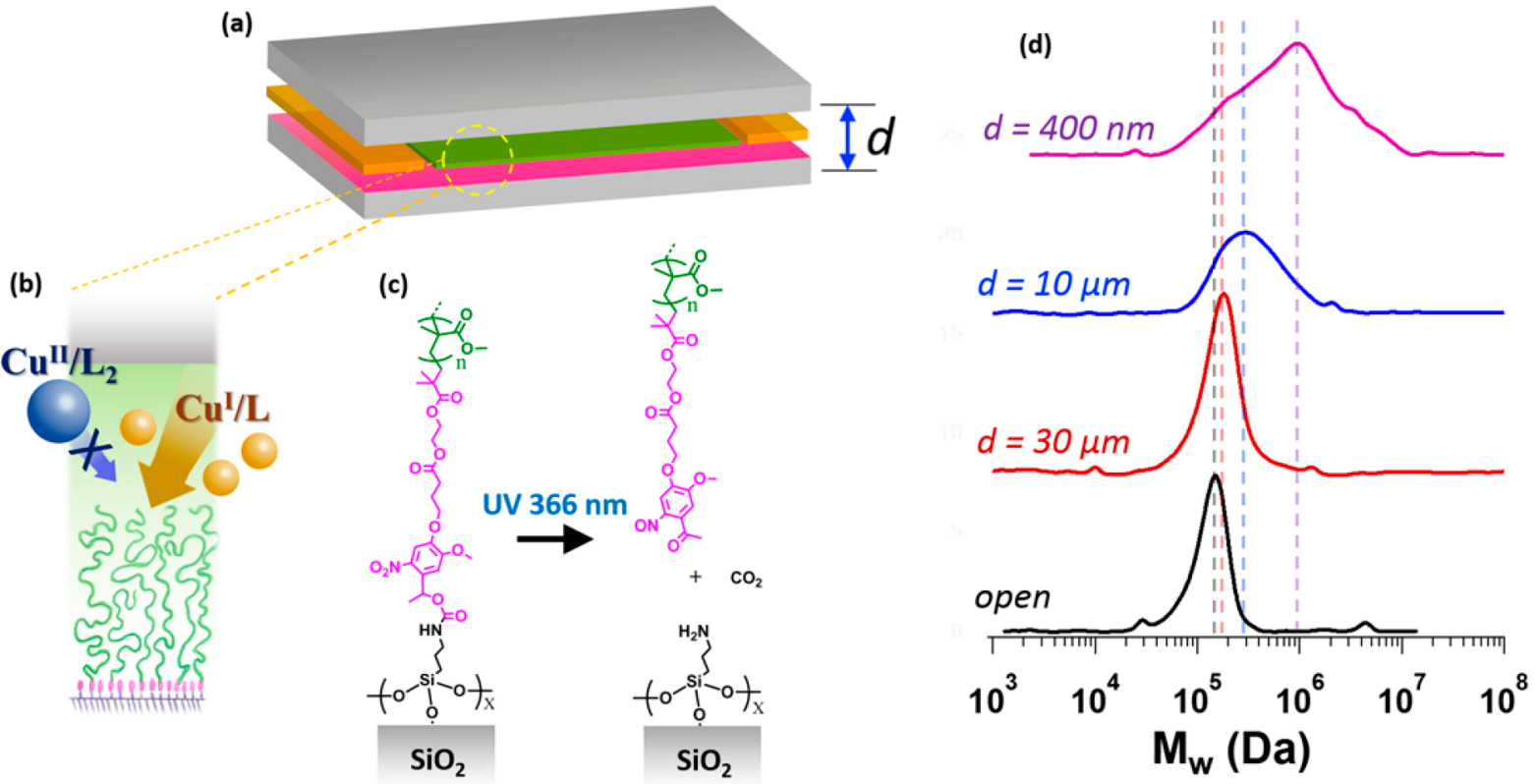

Figure 1. Spatially confined SI-ATRP reaction setups were fabricated by placing a PC-functionalized substrate at different distances $(d)$ from a bare $\mathrm{SiO}_{2}$ surface (confining surface) (a). A schematic depicting the polymer brush growing within the confined reaction volume is shown in (b). The chemical composition of the PC-based initiator layer is shown in (c). SEC elugrams of detached PMMA brushes synthesized under different confinements are shown in (d).

the fine measurement of the molar mass of polymer brushes generated by SI-ATRP in a confined space is a nontrivial task; and (iii) it is often difficult to define and control the extent of volume confinement within micro/nanostructured supports.

In order to circumvent these difficulties, we carried out SIATRP from a flat, initiator-functionalized $\mathrm{SiO}_{2}$ substrate, such that the dry thickness of the synthesized brush could be precisely measured by variable-angle spectroscopy ellipsometry (VASE). In addition, we applied a photocleavable SI-ATRP initiator, such that the molecular weight and polydispersity index (PDI) of the polymer brushes detached from the grafting surface could be conveniently measured by size-exclusion chromatography (SEC). ${ }^{24,25}$ Tuning of the volume confinement was achieved by positioning a bare silicon surface over the initiating substrate and varying the vertical distance between the two planes $(d)$ by means of vapor-deposited chromium patterns or thin, stainless-steel foil shims presenting uniform thickness (Figure 1), while the lateral boundaries of the volume are effectively defined by the rates of diffusion and kinetics of the reactions. In this way, the polymerization volume above the initiating surface can be varied between a highly confined space, with $d$ of a few hundred nanometers, to an open polymerization environment. SI-ATRP of methyl methacrylate (MMA) and lauryl methacrylate (LMA) showed remarkably faster kinetics upon increasing confinement (i.e., reducing $d$ ), generating brushes with nearly a 4-fold higher molar mass compared to the analogous grafts synthesized under an "open" polymerization volume, when the confining plane was positioned at a few hundred nanometers from the grafting surface. A significantly higher grafting rate is attributed to the slower diffusion of $\mathrm{Cu}^{\mathrm{II}}$ based deactivator species, which are bulkier than $\mathrm{Cu}^{\mathrm{I}}$-based activators, across viscous bulk polymerization mixtures, thus stimulating the formation of active chain ends at the brush growing front. The constrained-volume effect was especially evident when the viscosity of the polymerization mixtures was increased, as in the cases of bulk SI-ATRP of MMA performed in the presence of sacrificial initiator or that of LMA- a highmolar-mass methacrylate featuring an order-of-magnitude higher bulk viscosity than that of MMA. By combination of such conditions with highly confined volumes, ultrathick brushes can be generated within relatively short polymerization times.

The systematic analysis of confinement effects on SI-ATRP illustrates how their influence can be precisely modulated by simply tuning the polymerization volume, i.e., by a careful design of the reaction setup. Following a similar strategy, brush thickness gradients can be synthesized over a single substrate, demonstrating how an understanding of spatially confined SIATRP not only might be useful for multiscale fabrications but also can provide a synthetic tool for the precise structuring of polymeric platforms.

\section{EXPERIMENTAL SECTION}

Materials. Acetovanillone, ethyl 4-bromobutyrate, glacial acetic acid, acetic anhydride, $N, N$-dicyclohexylcarbodiimide (DCC), 4(dimethylamino)pyridine (DMAP), 2-hydroxyethyl 2-bromo-2methylpropanoate, sodium borohydride $\left(\mathrm{NaBH}_{4}\right)$, ammonium chloride $\left(\mathrm{NH}_{4} \mathrm{Cl}\right), N, N^{\prime}$-disuccinimidyl carbonate, and copper(II) bromide $\left(\mathrm{CuBr}_{2}\right)$ were purchased from Sigma-Aldrich (Germany) and used as received. 4,4'-Dinonyl-2,2'-bipyridyl (dNbpy) was purchased from ABCR Chemicals (Germany). Methyl methacrylate (MMA) and lauryl methacrylate (LMA) were purchased from Sigma-Aldrich and purified from inhibitors by passing them through basic alumina columns. $\mathrm{CuBr}$ was purchased from Sigma-Aldrich and purified by stirring overnight in glacial acetic acid, filtered, washed several times with diethyl ether and ethanol, and finally dried under vacuum.

NMR spectra were recorded using a Bruker AV300 spectrometer in suitable deuterated solvents using TMS as internal standard (Bruker, Germany). The molecular weights of the detached brushes were determined by a Viscotek size exclusion chromatograph (SEC, Malvern, Germany) using a Viscotek 302 TDA detector module (triple detector array comprising RI, light scattering, and viscosity detectors) with an additional UV detector (Viscotek 2500, $\lambda=254$ $\mathrm{nm}$ ). The separation of the polymers was achieved using two columns 
(PLGel Mix-B, PLGel Mix-C, from Agilent) and chloroform as eluent at $35{ }^{\circ} \mathrm{C}$ with a flow rate of $1.0 \mathrm{~mL} \mathrm{~min}{ }^{-1}$. The molecular weight of all the polymers was estimated by a universal calibration with polystyrene (PS) standards. The dry thickness of the brush films grafted from $\mathrm{SiO}_{2}$ substrates was measured using a variable-angle spectroscopic ellipsometer (VASE, M-2000F, LOT Oriel GmbH, Darmstadt, Germany) at an incident angle of $70^{\circ}$. The dry film thickness was measured by recording amplitude $(\Psi)$ and phase $(\Delta)$ components as a function of wavelength $(275-827 \mathrm{~nm})$ and applying a three-layer model featuring $\mathrm{Si}, \mathrm{SiO}_{2}$, and a Cauchy layer, using the known refractive indices of $\mathrm{Si}$ and $\mathrm{SiO}_{2}$ (software WVASE32, LOT Oriel GmbH, Darmstadt, Germany). ${ }^{26}$ A Cauchy model, $n=A+B / \lambda^{2}$, was used to describe the refractive index of the PMMA/PLMA films by means of two fitting parameters: offset $(A=1.45)$ and wavelength dispersion $(B=0.01)$.

Synthesis of ATRP Initiators. The photocleavable (PC) ATRP initiator was synthesized according to the previously described procedure $^{24,25}$ as reported in detail in the Supporting Information.

Fabrication of Chromium Patterns on $\mathrm{SiO}_{2}$ Substrates. Chromium patterns with a thickness varying between 200 and 900 $\mathrm{nm}$ were deposited on $\mathrm{SiO}_{2}$ substrates by vapor deposition using a BalTec MED 020 sputter coater equipped with a quartz crystal microbalance, operating at $3.8 \times 10^{-6}$ bar, using an additional $\mathrm{SiO}_{2}$ substrate as a mask to protect the central part of the substrate (Figure S2). Chromium deposition was carried out at a rate of $1 \mathrm{~nm} \mathrm{~s}^{-1}$ to ensure uniform metal film formation.

Functionalization of $\mathrm{SiO}_{2}$ Substrates with PC Initiator. Si (100) wafers were cut into three $4.0 \mathrm{~cm}^{2}$ pieces and cleaned with piranha solution $\left(\mathrm{H}_{2} \mathrm{O}_{2}: \mathrm{H}_{2} \mathrm{SO}_{4}=1: 3 \mathrm{v} / \mathrm{v}\right.$, room temperature, $20 \mathrm{~min}$; caution: piranha solution reacts violently with organic matter!), then washed extensively with Milli- $Q$ water, and dried under a stream of nitrogen. The substrates were subsequently functionalized with (3aminopropyl)triethoxysilane (APTES) by vapor deposition, subsequently washed with toluene and ethanol, and dried under a stream of nitrogen. PC initiator was reacted with the $\mathrm{NH}_{2}$-functionalized substrates by immersing them in a $10 \mathrm{~mL} 10 \mathrm{mg} \mathrm{mL}^{-1}$ solution of PC in dry THF overnight under $\mathrm{N}_{2}$ gas. Finally, the wafers were washed by sonication in THF and dried under a stream of nitrogen.

SI-ATRP of MMA and LMA. The SI-ATRP of MMA and LMA was carried out using a similar protocol, an exemplary procedure for MMA being reported here. A mixture of $0.164 \mathrm{~g}(0.4 \mathrm{mmol})$ of 4,4'dinonyl-2,2'-bipyridyl (dNbpy) and $35 \mathrm{~mL}(0.33 \mathrm{~mol})$ of MMA was degassed by four freeze-thaw cycles ( $15 \mathrm{~min}$ each) to remove oxygen. The degassed solution was transferred to a flask containing $26.8 \mathrm{mg}$ of $\mathrm{CuBr}(0.187 \mathrm{mmol})$ and $4.4 \mathrm{mg}$ of $\mathrm{CuBr}_{2}(0.019 \mathrm{mmol})$, which was kept under $\mathrm{N}_{2}$ gas. The mixture was subsequently stirred for $30 \mathrm{~min}$ and then transferred to a flask containing the PC-functionalized substrates and $24.0 \mathrm{mg}(0.048 \mathrm{mmol})$ of "sacrificial" free initiator 2((2-bromo-2-methylpropanoyl)oxy) ethyl4-(4-acetyl-2-methoxy-5nitrophenoxy)butanoate (see Supporting Information). The flask was later immersed into an oil bath set at $90{ }^{\circ} \mathrm{C}$, and the polymerization was carried out for $1 \mathrm{~h}$. Then, the functionalized substrates were taken out from the polymerization mixture, washed with chloroform, and subjected to Soxhlet extraction for $48 \mathrm{~h}$ in order to remove physisorbed polymer from the surface.

Spatially confined SI-ATRP was carried out using a polymerization mixture with the same composition (with and without sacrificial initiator) by covering the PC initiator-functionalized substrates with a bare $\mathrm{SiO}_{2}$ surface and varying the vertical distance $(d)$ between the two planes (degree of confinement) by $\mathrm{Cr}$ patterns that had been previously deposited at the sides of the initiating surface or by inserting stainless steel foils of different thicknesses $(10,30,50$, and $100 \mu \mathrm{m})$ between the two substrates (Figure S3 and Figure 1).

The effect of monomer concentration on confinement during SIATRP process was studied by using mixtures of MMA:anisole with ratios of 50:50 and 30:70 vol \%, while keeping the molar ratio of monomer to catalyst constant (MMA:dNbpy: $\mathrm{CuBr}: \mathrm{CuBr}_{2}=$ 1:0.82:0.47:0.05) compared to previous experiments. The detailed procedure for SI-ATRP using 50:50 vol \% MMA:anisole is as follows: a mixture of $17.5 \mathrm{~mL}$ of MMA, $17.5 \mathrm{~mL}$ of anisole, and $0.82 \mathrm{~g} \mathrm{(0.2}$ $\mathrm{mmol}$ ) of dNbpy was degassed four freeze-thaw cycles. The degassed solution was transferred to a flask containing $13.4 \mathrm{mg}(0.094 \mathrm{mmol})$ of $\mathrm{CuBr}$ and $2.2 \mathrm{mg}(0.010 \mathrm{mmol})$ of $\mathrm{CuBr}_{2}$ kept under $\mathrm{N}_{2}$ gas. This solution was stirred for $30 \mathrm{~min}$ and finally transferred into the flask containing the functionalized substrates and $24 \mathrm{mg}(0.048 \mathrm{mmol})$ of sacrificial initiator.

Photocleavage of Polymer Brushes. Detachment of polymer brushes was carried out following a previously reported method. 24,25 PC includes a dialkoxy nitrobenzyl group that can cleave under UV irradiation, generating amine, dioxide, and ketone groups (Figure 1). ${ }^{27}$ The photocleavage reaction of the initiator functions was carried out by exposing polymer-brush-functionalized substrates to UV light with $\lambda_{\max }=366 \mathrm{~nm}$ and a power density of $2.8 \mathrm{~mW} \mathrm{~cm}^{-2}$ for $1 \mathrm{~h}$. The irradiated substrates were washed three times with $1 \mathrm{~mL}$ of chloroform, and the obtained polymer solutions were concentrated until reaching $\sim 0.5 \mathrm{mg} \mathrm{mL}^{-1}$ and subsequently analyzed by SEC. The dry thickness of the polymer brushes was measured before and after UV irradiation, confirming a decrease in film thickness of $85 \%{ }^{25}$

\section{RESULTS AND DISCUSSION}

The influence of spatial confinement on SI-ATRP was investigated in the exemplary case of MMA, polymerized in bulk at $90{ }^{\circ} \mathrm{C}$ from self-assembled monolayers (SAMs) of the photocleavable 2-((2-bromo-2-methylpropanoyl)oxy)ethyl 4(4-(1-(((2,5-dioxopyrrolidin-1yl)oxy) carbonyl)oxy)ethyl)-2methoxy-5-nitrophenoxy)butanate (PC) initiator (Figure 1), and in the presence of a sacrificial initiator dissolved in the polymerization medium, which features a chemical composition comparable to the surface-immobilized analogue (see Supporting Information for details). The use of PC initiator to synthesize brushes and detach them from the grafting surface by UV irradiation is particularly advantageous when compared to other methods that involve the application of hazardous chemicals (e.g., HF treatment) and subsequent purification or long reaction times assuring the complete degrafting of brushes. The UV cleavage of PC anchors is relatively fast $(\sim 1 \mathrm{~h}$ of UV irradiation), allows the detachment of $85-90 \%$ of the brush film, and is reproducible and scalable to large substrates.

Variation of volume confinement above PC-functionalized substrates was achieved by placing an inert substrate at a fixed vertical distance $(d)$ from the initiating surface, by means of vapor-deposited $\mathrm{Cr}$ patterns and stainless steel foils of various thicknesses (Experimental Section). PC-modified substrates featuring different volume confinement (which we will simply express with the corresponding value of $d$ ) were incubated for $60 \mathrm{~min}$ in the reaction mixture, subsequently carefully washed by Soxhlet extraction, and finally analyzed by VASE to estimate the dry thickness of the generated PMMA brushes. The same brushes were detached from the grafting substrates by exploiting the cleavage of the dialkoxy nitrobenzyl groups within the PC functions upon irradiation with UV light $\left(\lambda_{\max }=\right.$ $366 \mathrm{~nm})^{25}$ and later analyzed by SEC (Figure 1).

As shown in Figure $2 \mathrm{a}$, at relatively large volume confinements $(d \geq 30 \mu \mathrm{m})$ the dry thickness of PMMA brushes remained nearly constant and corresponded well to the thickness of PMMA brushes synthesized under an open polymerization mixture $(d=\infty)$. In contrast, a significant increase in brush thickness was recorded for $d<20 \mu \mathrm{m}$, reaching $\sim 200 \mathrm{~nm}$ at the highest volume confinement tested in this study $(d=400 \mathrm{~nm})$, which is among the highest PMMA brush dry thicknesses ever obtained by controlled radical polymerization. The corresponding molar mass of the detached brushes exhibited a similar trend, with almost a 4-fold increment in $M_{\mathrm{n}}$ when $d$ was decreased from several tens of 
(a)

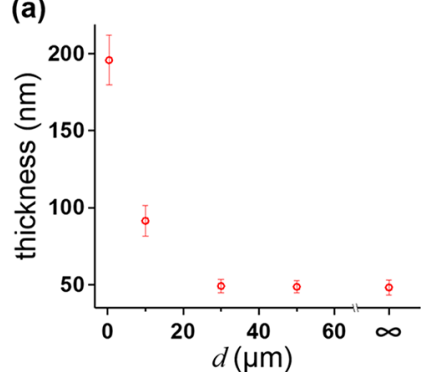

(b)

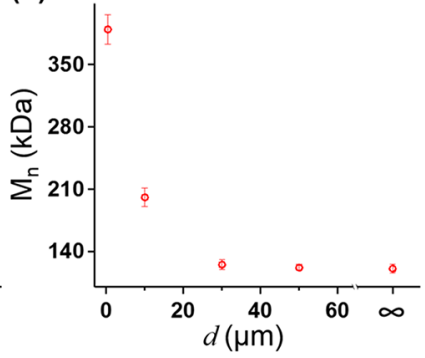

Figure 2. Dry thickness measured by VASE of PMMA brushes synthesized by $60 \mathrm{~min}$ of SI-ATRP under different degrees of confinement and in the presence of sacrificial initiator (a). The corresponding values of $M_{\mathrm{n}}$ for detached PMMA brushes were measured by SEC (b).

micrometers to $400 \mathrm{~nm}$ and a steep increase in tethered chain length for $d<20 \mu \mathrm{m}$ (Figure 2b and Table 1). The marked rise

Table 1. Dry Thickness, $M_{n}$, PDI, and Grafting Density of PMMA Brushes Synthesized under Different Degrees of Confinement by SI-ATRP

\begin{tabular}{|c|c|c|c|c|}
\hline $\begin{array}{c}\text { degree of } \\
\text { confinement }(d, \mu \mathrm{m})\end{array}$ & $\begin{array}{l}\text { thickness } \\
(\mathrm{nm})\end{array}$ & $\begin{array}{c}M_{\mathrm{n}} \\
(\mathrm{kDa})\end{array}$ & PDI & $\begin{array}{l}\text { grafting density } \\
\text { (chains } \mathrm{nm}^{-2} \text { ) }\end{array}$ \\
\hline 0.4 & $196 \pm 16$ & 390 & 2.20 & 0.35 \\
\hline 10 & $92 \pm 10$ & 200 & 1.59 & 0.31 \\
\hline 30 & $49 \pm 4$ & 125 & 1.23 & 0.27 \\
\hline 50 & $49 \pm 3$ & 122 & 1.12 & 0.28 \\
\hline open $(\infty)$ & $48 \pm 5$ & 121 & 1.11 & 0.28 \\
\hline
\end{tabular}

of $M_{\mathrm{n}}$ by PMMA brushes synthesized under confined volumes was also accompanied by an increment in their PDI, which grew from $\sim 1.1$ when SI-ATRP was performed under an open medium to $\sim 1.5$ for $d=10 \mu \mathrm{m}$, finally reaching $\sim 2.2$ at the lowest $d$ value of $400 \mathrm{~nm}$ (Table 1).

These results collectively suggest that SI-ATRP of MMA proceeded at a higher rate within highly confined volumes, generating thicker films after a given polymerization time. The increment of molar mass by PMMA brushes with increasing confinement was also accompanied by an increase in the PDI, suggesting a progressive loss of control over the polymerization process when the reaction volume is decreased. The measured grafting density $(\sigma)$ remained nearly constant, irrespective of confinement, with just a slight increment from 0.28 chains $\mathrm{nm}^{-2}$, for $d=\infty$, to 0.35 chains $\mathrm{nm}^{-2}$, for $d=400 \mathrm{~nm}$. This small variation could be explained by considering that the effect of the higher PDI of brushes synthesized under increasing confinement was not taken into account in the estimate of $\sigma$.

We recently reported how irreversible radical recombination reactions between growing chains in the case of surfaceinitiated $\mathrm{Cu}(0)$-mediated radical polymerization (SI-CuCRP) performed in the presence of a spatially confining $\mathrm{Cu}(0)$-coated surface, significantly affected the grafting density of the synthesized brushes. ${ }^{28}$ However, in the present study, an increment of volume confinement during SI-ATRP substantially affected the molar mass of the synthesized brushes and their PDI, while the brush architecture remained unaltered overall. The following experiments have provided a rationale as to how the SI-ATRP mechanism was influenced by high degrees of volume confinement.

In the absence of sacrificial initiator, the SI-ATRP of MMA performed under comparable experimental conditions showed

analogous results, the thickness of the PMMA brushes steadily increasing when $d$ was decreased below $10 \mu \mathrm{m}$. However, the dry thickness of PMMA brushes was in all cases nearly an order of magnitude lower than that obtained by complementing the polymerization mixture with sacrificial initiator (Figure 3a).
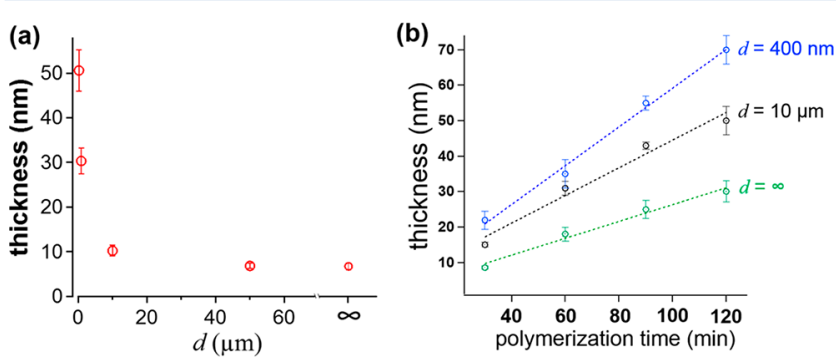

Figure 3. Dry thickness obtained by VASE of PMMA brushes synthesized by $60 \mathrm{~min}$ of SI-ATRP without sacrificial initiator under different degrees of volume confinement (a). The corresponding brush thickening rates, recorded over $120 \mathrm{~min}$ of SI-ATRP (b).

This result could be due to a loss of control and the consequent occurrence of radical termination/transfer reactions caused by the reduced concentration of deactivator $\mathrm{Cu}^{\text {II }}$-based species. $^{29-32}$ Yet, grafting-kinetics experiments performed applying the same polymerization conditions and monitoring the brush thickening rates over $2 \mathrm{~h}$ of reaction did not show any signs of termination, linear thickness-vs-time profiles suggesting the controlled grafting of PMMA chains from the surface (Figure $3 \mathrm{~b}$ ). Interestingly, the brush thickening rates reported in Figure $3 \mathrm{~b}$ highlight how SI-ATRP of MMA under confinement showed higher growth rates when compared to an open process performed under the same reaction conditions, confirming the faster kinetics provided by the presence of a confining surface placed in the proximity of the grafting plane.

This evidence suggests that the faster kinetics for SI-ATRP of MMA and thus the significantly higher thickness (molar mass) of PMMA brushes synthesized under confinement were probably due to viscosity-induced effects, altering the polymerization mechanism within a spatially constrained reaction volume. ${ }^{33}$ In the presence of sacrificial initiator, the viscosity of the polymerization solution steadily increased during the course of the reaction, due to the free polymer forming in the medium in contact with the growing brushes. Simultaneously, the equilibria at the growing brush front determined the polymerization rate and ultimately the brush parameters. At a given polymerization time, the concentration of active radicals at the above-mentioned front is kept low, as determined by the ATRP equilibrium constants, with a large majority of tethered chainends being in their dormant state. ${ }^{34}$ Upon the progressive increase in viscosity of the polymerization medium, the $\mathrm{Cu}^{\mathrm{I}}$ based activator species could diffuse more readily to the brush front than the bulkier $\mathrm{Cu}^{\mathrm{II}}$-based deactivators, ${ }^{35}$ which were significantly hindered within a restricted reaction volume. This phenomenon, coupled with the low rate of irreversible termination, led to a higher surface concentration of active chain-ends compared to the open SI-ATRP, generating highmolar-mass grafts within a relatively short polymerization time. Diffusion-controlled deactivation ${ }^{36-38}$ was enhanced when sacrificial initiators were added to the polymerization mixture, playing a similar role to the well-known Trommsdorff effect, which brings about an increase in the polymerization rate for free-radical polymerization at high conversions. ${ }^{37,38}$ 

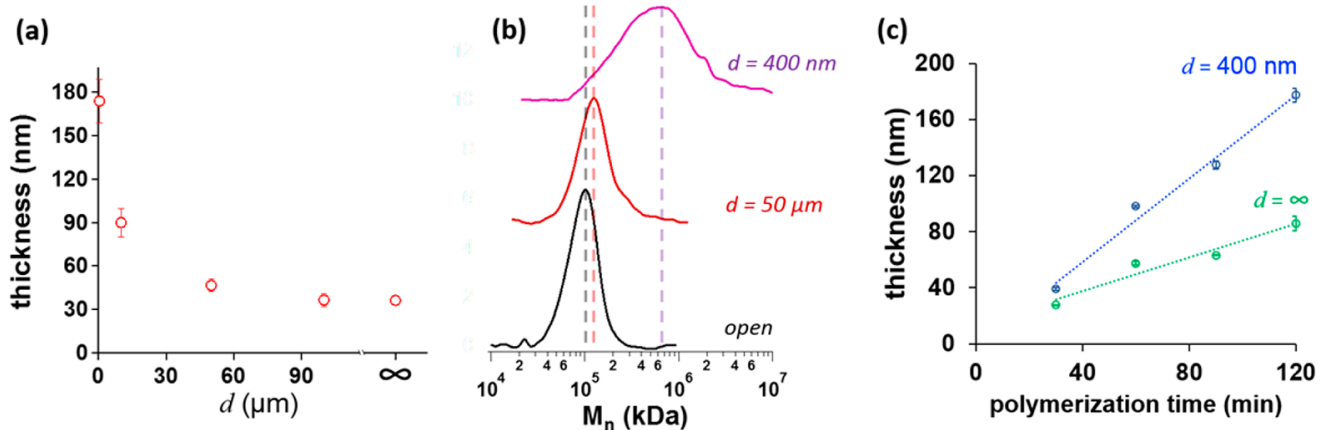

Figure 4. (a) Dry VASE thickness of PLMA brushes synthesized by $60 \mathrm{~min}$ of SI-ATRP within different confined volumes. (b) GPC traces of detached PLMA brushes synthesized by SI-ATRP within differently confined volumes. For $d=\infty: M_{\mathrm{n}}=97000 \mathrm{Da}, M_{w}=101000 \mathrm{Da}$, PDI $=1.04$. For $d=50 \mu \mathrm{m}, M_{\mathrm{n}}=113000 \mathrm{Da}, M_{w}=147000 \mathrm{Da}$, PDI $=1.30$. For $d=400 \mathrm{~nm}, M_{\mathrm{n}}=415000 \mathrm{Da}, M_{w}=1300000 \mathrm{Da}$, PDI = 3.13. (c) PLMA brush thickening rates recorded by ex situ VASE over $120 \mathrm{~min}$ of polymerization.

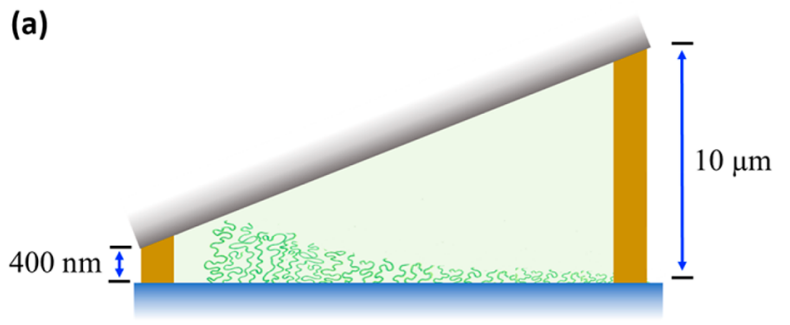

(b)

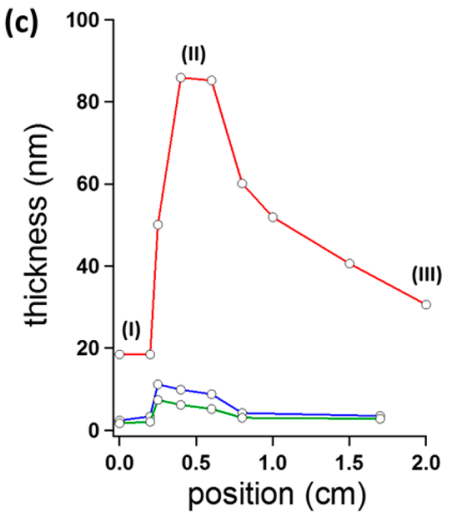

Figure 5. SI-ATRP setup applied for the fabrication of PMMA brush gradients exploiting a variable volume confinement (a). The subsequently obtained PMMA brush gradient (b) presented a variation of dry thickness across the substrates, as recorded by VASE measurements (c). The steepness of the brush gradient can be tuned by diluting MMA with anisole during the SI-ATRP: bulk (red trace), 50:50 vol \% (blue trace), and 70:30 vol \% (green trace) anisole:MMA.

It is noteworthy that a faster rate of SI-ATRP, reminiscent of that observed here, was previously observed when the grafting of polystyrene (PS), PMMA, and block copolymer brushes was performed from nanoclay sheets or graphene layers. ${ }^{41-44}$ Although in most of these works the faster ATRP kinetics were ascribed to the active involvement of the inorganic/ organic nanostructures in the polymerization process, Behling et al., while studying the influence of initiator coverage on the SI-ATRP of styrene within nanoclay tactoids, suggested that the higher polymerization rate could be due to a slower diffusion of deactivator species within these highly confined interstitial layers. ${ }^{33}$ In the present study, we systematically demonstrate that within confined reaction volumes SI-ATRP produces ultrathick PMMA brushes, while the polymer grafting rate can be precisely tuned by varying the degree of confinement. It is also relevant that faster kinetics led to a progressive increase in PDI (Table 1). An increment of grafted-chain polydispersity upon a decrease in $d$ was likely due to an inhomogeneous growth of PMMA brushes, not all the grafts being subjected to uniform activation/deactivation at the brush front when this is facing an increasingly confined space. Under these conditions, a large number of propagating polymer chains in their active state are likely to experience radical recombination and transfer reactions, which contribute to the broadening of the graftedchain-length distribution.

In order to confirm that viscosity-controlled deactivation determines the grafting kinetics within confined reaction volumes, we performed SI-ATRP of LMA, this monomer having a reactivity comparable to MMA although with an orderof-magnitude higher bulk viscosity $\left(\eta_{\mathrm{LMA}}=6.0 \mathrm{mPa} \cdot \mathrm{s}\right.$ compared to $\eta_{\text {MMA }}=0.5 \mathrm{mPa} \cdot \mathrm{s}$ at $\left.20{ }^{\circ} \mathrm{C}^{43}\right)$. PLMA brushes were synthesized by SI-ATRP under comparable polymerization conditions to those applied for PMMA analogues in the absence of sacrificial initiator and applying different degrees of volume confinement, with $d$ ranging from a few tens of micrometers to $400 \mathrm{~nm}$ (see Experimental Section for details). Remarkably, SI-ATRP of LMA generated much thicker brushes compared to PMMA grafted under similar conditions and under comparable confinement. Namely, $\sim 170 \mathrm{~nm}$ thick PLMA brushes were synthesized within $60 \mathrm{~min}$ of polymerization with $d=400 \mathrm{~nm}$ (Figure 4a), corresponding to a nearly 4-fold increase of $M_{n}$ compared to that resulting from an open polymerization mixture (Figure $4 \mathrm{~b}$ ). In addition, also in the case of PLMA, brush PDI progressively increased with increasing confinement, confirming the loss of control already observed in the case of PMMA brushes. As displayed in Figure $4 \mathrm{c}$, the synthesis of thicker PLMA brushes was correlated to faster brush growth. Hence, the viscous polymerization mixture generated by LMA strongly altered the SI-ATRP kinetics, in a similar way to that previously observed for PMMA grafted in the presence of soluble free initiator.

The faster grafting kinetics of SI-ATRP under confinement might have a significant impact on systems involving polymer growth within nano/microstructured environments and can be 
exploited to structure polymer interfaces with high precision and reproducibility. Although efforts have been increasingly spent by us and others in proposing accessible methods for the fabrication of nanostructured polymer brushes ${ }^{46-59}$ and brush gradients $^{60-63}$ by SI-CRP, these often rely on sophisticated and sometimes tedious multistep, surface-modification protocols, which frequently suffer from a lack of reproducibility. An experimentally simple demonstration of the intrinsic applicability of confined SI-ATRP to synthesize nanostructured polymeric platforms is provided by the fabrication of polymerbrush gradients, utilizing an initiating substrate presenting a confining surface, positioned at a variable distance ("tilted" confining plane, as schematically depicted in Figure 5a). As highlighted in Figure 5, two spacers of thickness $400 \mathrm{~nm}$ and 10 $\mu \mathrm{m}$ were placed at each end of an $\sim 2 \mathrm{~cm}$ long, initiatorfunctionalized $\mathrm{SiO}_{2}$ substrate. This was covered by a bare $\mathrm{SiO}_{2}$ surface (confining plane), thus generating a continuous variation of confinement degree across the initiating surface (Figure 5a). SI-ATRP of MMA performed within this setup produced a relatively steep brush thickness gradient following 1 $\mathrm{h}$ of reaction, with the dry PMMA brush thickness ranging from almost $90 \mathrm{~nm}$ (at high confinement) to $\sim 30 \mathrm{~nm}$ (at low confinement) (Figures $5 \mathrm{~b}$ and $5 \mathrm{c}$ ) across $\sim 1.5 \mathrm{~cm}$.

As a further confirmation of the synergistic action of increased viscosity and volume confinement to yield higher brush-growth rates, we fabricated analogous brush gradients by applying diluted polymerization mixtures, comprising 50:50 and 70:30 vol \% anisole:MMA mixtures. As shown in Figure 5c, PMMA brush gradients presenting much less variation of thickness across the substrates were obtained. These results indicated that a reduction of viscosity within the polymerization mixture markedly reduced the effect of volume confinement, producing just a slight increase of PMMA brush thickness when the inert plane was in close proximity to the grafting surface. Alternatively, the modulation of brush-gradient morphology by dilution of the polymerization mixtures demonstrates how brush surfaces with different gradient-like structures could be easily generated by tuning the extent of confinement and the composition of the reaction solution.

\section{CONCLUSIONS}

We have systematically investigated the SI-ATRP of common methacrylates within confined environments, demonstrating that a progressive increment of the polymerization rates with increasing confinement is due to a hampering of radical deactivation within viscous and spatially hindered reaction volumes. Photocleavable ATRP-initiator-functionalized surfaces, allowing the convenient detachment and SEC analysis of the synthesized brushes, were positioned at a variable distance (d) from a confining, inert surface by micropatterned or thinfoil spacers. At a high degree of volume confinement with $d=$ $400 \mathrm{~nm}$ and in the presence of sacrificial initiator, the SI-ATRP of MMA generated a 4-fold increase in the brush molar mass compared to that obtained by applying an open polymerization setup. A similar increment of brush growth-rate could be observed for the more viscous LMA, in the absence of free initiator, confirming how faster grafting kinetics resulted from the progressive hindering of bulkier $\mathrm{Cu}^{\mathrm{II}}$-based deactivators toward the brush growing front when the polymerization was performed within a highly constrained reaction volume.

Besides being fundamentally relevant for the synthesis of polymer brushes from micro- and nanostructured platforms, confined SI-ATRP could be a powerful tool for fabricating brush gradients with high precision, by simply varying the distance between the initiating and the confining surfaces within a single polymerization setup.

These findings highlight how fine spatial control over a robust and reliable polymerization process such as ATRP can translate into a highly accessible tool for tuning the properties of polymer brushes, finally providing an attractive approach that could be widely applied in polymer and materials science.

\section{ASSOCIATED CONTENT}

\section{S Supporting Information}

The Supporting Information is available free of charge on the ACS Publications website at DOI: 10.1021/acs.macromol.7b00919.

Synthesis of PC initiator and the methods for fabricating the confined SI-ATRP setups (PDF)

\section{AUTHOR INFORMATION}

\section{Corresponding Authors}

*E-mail: edmondo.benetti@mat.ethz.ch (E.M.B.).

*E-mail: nspencer@ethz.ch (N.D.S.).

\section{ORCID $\odot$}

Edmondo M. Benetti: 0000-0002-5657-5714

Nicholas D. Spencer: 0000-0002-7873-7905

\section{Funding}

This project received funding from the European Research Council (ERC) under the European Union's Horizon 2020 research and innovation program (Grant Agreement No. 669562). E.M.B. acknowledges financial support from the Swiss National Science Foundation (SNSF "Ambizione" PZ00P2-148156).

\section{Notes}

The authors declare no competing financial interest.

\section{REFERENCES}

(1) Milner, S. T. Polymer Brushes. Science 1991, 251, 905-914.

(2) Zhao, B.; Brittain, W. J. Polymer brushes: surface-immobilized macromolecules. Prog. Polym. Sci. 2000, 25, 677-710.

(3) Zoppe, J. O.; Ataman, N. C.; Mocny, P.; Wang, J.; Moraes, J.; Klok, H. A. Surface-Initiated Controlled Radical Polymerization: Stateof-the-Art, Opportunities, and Challenges in Surface and Interface Engineering with Polymer Brushes. Chem. Rev. 2017, 117, 4667-4667.

(4) Couet, J.; Jeyaprakash, J. D.; Samuel, S.; Kopyshev, A.; Santer, S.; Biesalski, M. Peptide-polymer hybrid nanotubes. Angew. Chem., Int. Ed. 2005, 44, 3297-3301.

(5) Banerjee, S.; Paira, T. K.; Kotal, A.; Mandal, T. K. SurfaceConfined Atom Transfer Radical Polymerization from Sacrificial Mesoporous Silica Nanospheres for Preparing Mesoporous Polymer/ Carbon Nanospheres with Faithful Shape Replication: Functional Mesoporous Materials. Adv. Funct. Mater. 2012, 22, 4751-4762.

(6) Yah, W. O.; Xu, H.; Soejima, H.; Ma, W.; Lvov, Y.; Takahara, A. Biomimetic Dopamine Derivative for Selective Polymer Modification of Halloysite Nanotube Lumen. J. Am. Chem. Soc. 2012, 134, 1213412137.

(7) Ito, Y.; Park, Y. S.; Imanishi, Y. Visualization of critical pHcontrolled gating of a porous membrane grafted with polyelectrolyte brushes. J. Am. Chem. Soc. 1997, 119, 2739-2740.

(8) Yang, B.; Yang, W. T. Thermo-sensitive switching membranes regulated by pore-covering polymer brushes. J. Membr. Sci. 2003, 218, 247-255.

(9) Balachandra, A. M.; Baker, G. L.; Bruening, M. L. Preparation of composite membranes by atom transfer radical polymerization initiated from a porous support. J. Membr. Sci. 2003, 227, 1-14. 
(10) Jain, P.; Sun, L.; Dai, J. H.; Baker, G. L.; Bruening, M. L. Highcapacity purification of his-tagged proteins by affinity membranes containing functionalized polymer brushes. Biomacromolecules 2007, 8, 3102-3107.

(11) Bruening, M. L.; Dotzauer, D. M.; Jain, P.; Ouyang, L.; Baker, G. L. Creation of functional membranes using polyelectrolyte multilayers and polymer brushes. Langmuir 2008, 24, 7663-7673.

(12) de Groot, G. W.; Santonicola, M. G.; Sugihara, K.; Zambelli, T.; Reimhult, E.; Voros, J.; Vancso, G. J. Switching Transport through Nanopores with $\mathrm{pH}$-Responsive Polymer Brushes for Controlled Ion Permeability. ACS Appl. Mater. Interfaces 2013, 5, 1400-1407.

(13) Nie, C. X.; Ma, L.; Xia, Y.; He, C.; Deng, J.; Wang, L. R.; Cheng, C.; Sun, S. D.; Zhao, C. S. Novel heparin-mimicking polymer brush grafted carbon nanotube/PES composite membranes for safe and efficient blood purification. J. Membr. Sci. 2015, 475, 455-468.

(14) Keating, J. J.; Imbrogno, J.; Belfort, G. Polymer Brushes for Membrane Separations: A Review. ACS Appl. Mater. Interfaces 2016, 8, 28383-28399.

(15) Calvo, A.; Yameen, B.; Williams, F. J.; Soler-Illia, G. J. A. A.; Azzaroni, O. Mesoporous Films and Polymer Brushes Helping Each Other To Modulate Ionic Transport in Nanoconfined Environments. An Interesting Example of Synergism in Functional Hybrid Assemblies. J. Am. Chem. Soc. 2009, 131, 10866-10868.

(16) Huang, X. Y.; Doneski, L. J.; Wirth, M. J. Surface-confined living radical polymerization for coatings in capillary electrophoresis. Anal. Chem. 1998, 70, 4023-4029.

(17) Xu, C.; Wu, T.; Drain, C. M.; Batteas, J. D.; Beers, K. L. Microchannel confined surface-initiated polymerization. Macromolecules 2005, 38, 6-8.

(18) Costantini, F.; Benetti, E. M.; Tiggelaar, R. M.; Gardeniers, H. J. G. E.; Reinhoudt, D. N.; Huskens, J.; Vancso, G. J.; Verboom, W. A Brush-Gel/Metal-Nanoparticle Hybrid Film as an Efficient Supported Catalyst in Glass Microreactors. Chem. - Eur. J. 2010, 16, 1240612411.

(19) Costantini, F.; Benetti, E. M.; Reinhoudt, D. N.; Huskens, J.; Vancso, G. J.; Verboom, W. Enzyme-functionalized polymer brush films on the inner wall of silicon-glass microreactors with tunable biocatalytic activity. Lab Chip 2010, 10, 3407-3412.

(20) Yu, Q.; Ista, L. K.; Gu, R. P.; Zauscher, S.; Lopez, G. P. Nanopatterned polymer brushes: conformation, fabrication and applications. Nanoscale 2016, 8, 680-700.

(21) Matyjaszewski, K.; Tsarevsky, N. V. Nanostructured functional materials prepared by atom transfer radical polymerization. Nat. Chem. 2009, 1, 276-288.

(22) Pyun, J.; Jia, S. J.; Kowalewski, T.; Patterson, G. D.; Matyjaszewski, K. Synthesis and characterization of organic/inorganic hybrid nanoparticles: Kinetics of surface-initiated atom transfer radical polymerization and morphology of hybrid nanoparticle ultrathin films. Macromolecules 2003, 36, 5094-5104.

(23) Khabibullin, A.; Mastan, E.; Matyjaszewski, K.; Zhu, S. P. Surface-Initiated Atom Transfer Radical Polymerization. Adv. Polym. Sci. 2015, 270, 29-76.

(24) Kang, C. J.; Ramakrishna, S. N.; Nelson, A.; Cremmel, C. V. M; vom Stein, H.; Spencer, N. D.; Isa, L.; Benetti, E. M. Ultrathin, freestanding, stimuli-responsive, porous membranes from polymer hydrogel-brushes. Nanoscale 2015, 7, 13017-13025.

(25) Kang, C. J.; Crockett, R. M.; Spencer, N. D. Molecular-Weight Determination of Polymer Brushes Generated by SI-ATRP on Flat Surfaces. Macromolecules 2014, 47, 269-275.

(26) Tompkins, H.; Irene, E. A. Handbook of Ellipsometry; Springer: Berlin, 2005.

(27) Papageorgiou, G.; Corrie, J. E. T. Synthesis and properties of carbamoyl derivatives of photolabile benzoins. Tetrahedron 1997, 53, 3917-3932.

(28) Dehghani, E. S.; Du, Y. H.; Zhang, T.; Ramakrishna, S. N.; Spencer, N. D.; Jordan, R.; Benetti, E. M. Fabrication and Interfacial Properties of Polymer Brush Gradients by Surface-Initiated $\mathrm{Cu}(0)$ Mediated Controlled Radical Polymerization. Macromolecules 2017, 50, 2436-2446.
(29) Matyjaszewski, K.; Miller, P. J.; Shukla, N.; Immaraporn, B.; Gelman, A.; Luokala, B. B.; Siclovan, T. M.; Kickelbick, G.; Vallant, T.; Hoffmann, H.; Pakula, T. Polymers at interfaces: Using atom transfer radical polymerization in the controlled growth of homopolymers and block copolymers from silicon surfaces in the absence of untethered sacrificial initiator. Macromolecules 1999, 32, 8716-8724.

(30) Kim, J. B.; Huang, W. X.; Miller, M. D.; Baker, G. L.; Bruening, M. L. Kinetics of surface-initiated atom transfer radical polymerization. J. Polym. Sci., Part A: Polym. Chem. 2003, 41, 386-394.

(31) Cheng, N.; Azzaroni, O.; Moya, S.; Huck, W. T. S. The effect of $[\mathrm{Cu}-\mathrm{I}] /[\mathrm{Cu}-\mathrm{II}]$ ratio on the kinetics and conformation of polyelectrolyte brushes by atom transfer radical polymerization. Macromol. Rapid Commun. 2006, 27, 1632-1636.

(32) Jeyaprakash, J. D.; Samuel, S.; Dhamodharan, R.; Ruhe, J. Polymer brushes via ATRP: Role of activator and deactivator in the surface-initiated ATRP of styrene on planar substrates. Macromol. Rapid Commun. 2002, 23, 277-281.

(33) Behling, R. E.; Williams, B. A.; Staade, B. L.; Wolf, L. M.; Cochran, E. W. Influence of Graft Density on Kinetics of SurfaceInitiated ATRP of Polystyrene from Montmorillonite. Macromolecules 2009, 42, 1867-1872.

(34) Wang, J. S.; Matyjaszewski, K. Controlled Living Radical Polymerization - Atom-Transfer Radical Polymerization in the Presence of Transition-Metal Complexes. J. Am. Chem. Soc. 1995, $117,5614-5615$.

(35) Rabea, A. M.; Zhu, S. P. Modeling the Influence of DiffusionControlled Reactions and Residual Termination and Deactivation on the Rate and Control of Bulk ATRP at High Conversions. Polymers 2015, 7, 819-835.

(36) Wang, A. R.; Zhu, S. P. ESR study on diffusion-controlled atom transfer radical polymerization of methyl methacrylate and ethylene glycol dimethacrylate. Macromolecules 2002, 35, 9926-9933.

(37) Rabea, A. M.; Zhu, S. P. Controlled Radical Polymerization at High Conversion: Bulk ICAR ATRP of Methyl Methacrylate. Ind. Eng. Chem. Res. 2014, 53, 3472-3477.

(38) Rabea, A. M.; Zhu, S. P. Achieving High-Conversion Bulk ATRP with Good Livingness and Well Controlled by Design and Optimization of Polymerization Temperature Profile. Macromol. React. Eng. 2014, 8, 771-776.

(39) Dvornic, P. R.; Jacovic, M. S. The Viscosity Effect on AutoAcceleration of the Rate of Free-Radical Polymerization. Polym. Eng. Sci. 1981, 21, 792-796.

(40) Russell, G. T.; Napper, D. H.; Gilbert, R. G. Termination in Free-Radical Polymerizing Systems at High Conversion. Macromolecules 1988, 21, 2133-2140.

(41) Singha, N. K.; Kavitha, A.; Haloi, D. J.; Mandal, P.; Janke, A.; Jehnichen, D.; Komber, H.; Voit, B. Effect of Nanoclay on in situ Preparation of "All Acrylate" ABA Triblock Copolymers via ATRP and Their Morphology. Macromol. Chem. Phys. 2012, 213, 2034-2043.

(42) Roghani-Mamaqani, H.; Haddadi-Asl, V.; Khezri, K.; SalamiKalajahi, M.; Najafi, M. Kinetic Study of Styrene Atom Transfer Radical Polymerization From Hydroxyl Groups of Graphene Nanoplatelets: Heterogeneities in Chains and Graft Densities. Polym. Eng. Sci. 2015, 55, 1720-1732.

(43) Roghani-Mamaqani, H.; Haddadi-Asl, V.; Khezri, K.; SalamiKalajahi, M. Polystyrene-grafted graphene nanoplatelets with various graft densities by atom transfer radical polymerization from the edge carboxyl groups. RSC Adv. 2014, 4, 24439-24452.

(44) Roghani-Mamaqani, H. Surface-initiated ATRP of styrene from epoxy groups of graphene nanolayers: twofold polystyrene chains and various graft densities. RSC Adv. 2015, 5, 53357-53368.

(45) Brandrup, J.; Immergut, H. E.; Grulke, E. A. Polymer Handbook, 4th ed.; J. Wiley \& Sons: New York, 1999.

(46) von Werne, T. A.; Germack, D. S.; Hagberg, E. C.; Sheares, V. V.; Hawker, C. J.; Carter, K. R. A versatile method for tuning the chemistry and size of nanoscopic features by living free radical polymerization. J. Am. Chem. Soc. 2003, 125, 3831-3838. 
(47) Liu, X. G.; Guo, S. W.; Mirkin, C. A. Surface and site-specific ring-opening metathesis polymerization initiated by dip-pen nanolithography. Angew. Chem., Int. Ed. 2003, 42, 4785-4789.

(48) Kaholek, M.; Lee, W. K.; LaMattina, B.; Caster, K. C.; Zauscher, $\mathrm{S}$. Fabrication of stimulus-responsive nanopatterned polymer brushes by scanning-probe lithography. Nano Lett. 2004, 4, 373-376.

(49) Ahn, S. J.; Kaholek, M.; Lee, W. K.; LaMattina, B.; LaBean, T. $\mathrm{H}$.; Zauscher, S. Surface-initiated polymerization on nanopatterns fabricated by electron-beam lithography. Adv. Mater. 2004, 16, 21412145.

(50) Lee, W. K.; Caster, K. C.; Kim, J.; Zauscher, S. Nanopatterned polymer brushes by combining AFM anodization lithography with ring-opening metathesis polymerization in the liquid and vapor phase. Small 2006, 2, 848-853.

(51) Schmelmer, U.; Paul, A.; Kuller, A.; Steenackers, M.; Ulman, A.; Grunze, M.; Golzhauser, A.; Jordan, R. Nanostructured polymer brushes. Small 2007, 3, 459-465.

(52) Zapotoczny, S.; Benetti, E. M.; Vancso, G. J. Preparation and characterization of macromolecular "hedge" brushes grafted from $\mathrm{Au}$ nanowires. J. Mater. Chem. 2007, 17, 3293-3296.

(53) Benetti, E. M.; Chung, H. J.; Vancso, G. J. pH Responsive Polymeric Brush Nanostructures: Preparation and Characterization by Scanning Probe Oxidation and Surface Initiated Polymerization. Macromol. Rapid Commun. 2009, 30, 411-417.

(54) Benetti, E. M.; Acikgoz, C.; Sui, X. F.; Vratzov, B.; Hempenius, M. A.; Huskens, J.; Vancso, G. J. Nanostructured Polymer Brushes by UV-Assisted Imprint Lithography and Surface-Initiated Polymerization for Biological Functions. Adv. Funct. Mater. 2011, 21, 2088-2095.

(55) Hutter, N. A.; Steenackers, M.; Reitinger, A.; Williams, O. A.; Garrido, J. A.; Jordan, R. Nanostructured polymer brushes and protein density gradients on diamond by carbon templating. Soft Matter 2011, 7, 4861-4867.

(56) Chen, T.; Amin, I.; Jordan, R. Patterned polymer brushes. Chem. Soc. Rev. 2012, 41, 3280-3296.

(57) Yu, Q.; Ista, L. K.; Gu, R. P.; Zauscher, S.; Lopez, G. P. Nanopatterned polymer brushes: conformation, fabrication and applications. Nanoscale 2016, 8, 680-700.

(58) Parry, A. V. S.; Straub, A. J.; Villar-Alvarez, E. M.; Phuengphol, T.; Nicoll, J. E. R.; Lim, W. K. X.; Jordan, L. M.; Moore, K. L.; Taboada, P.; Yeates, S. G.; Edmondson, S. Submicron Patterning of Polymer Brushes: An Unexpected Discovery from Inkjet Printing of Polyelectrolyte Macroinitiators. J. Am. Chem. Soc. 2016, 138, 90099012.

(59) Nawroth, J. F.; Neisser, C.; Erbe, A.; Jordan, R. Nanopatterned polymer brushes by reactive writing. Nanoscale 2016, 8, 7513-7522.

(60) Bhat, R. R.; Tomlinson, M. R.; Wu, T.; Genzer, J. Surfacegrafted polymer gradients: Formation, characterization, and applications. Adv. Polym. Sci. 2006, 198, 51-124.

(61) Schuh, C.; Santer, S.; Prucker, O.; Ruhe, J. Polymer Brushes with Nanometer-Scale Gradients. Adv. Mater. 2009, 21, 4706-4710.

(62) Gunnewiek, M. K.; Ramakrishna, S. N.; Di Luca, A.; Vancso, G. J.; Moroni, L.; Benetti, E. M. Stem-Cells Clinging by a Thread: AFM Measure of Polymer-Brush Lateral Deformation. Adv. Mater. Interfaces 2016, 3, 1500456.

(63) Benetti, E. M.; Gunnewiek, M. K.; van Blitterswijk, C. A.; Vancso, G. J.; Moroni, L. Mimicking natural cell environments: design, fabrication and application of bio-chemical gradients on polymeric biomaterial substrates. J. Mater. Chem. B 2016, 4, 4244-4257. 